\title{
EULAR 2021 updated viewpoints on SARS-CoV-2 vaccination in patients with RMDs: a guidance to answer patients' questions
}

\author{
Johannes WJ Bijlsma (1) , On behalf of the EULAR COVID-19 Task Force
}

Handling editor David S Pisetsky

\section{Correspondence to}

Dr Johannes WJ Bijlsma, Department of Rheumatology and Clinical Immunology, University Medical Center Utrecht, Utrecht, The Netherlands; j.w.j.bijlsma@gmail.com

Received 5 December 2021 Accepted 19 January 2022

Check for updates

(C) Author(s) (or their employer(s)) 2022. No commercial re-use. See rights and permissions. Published by BMJ.

To cite: Bijlsma JWJ, Ann Rheum Dis Epub ahead of print: [please include Day Month Year]. doi:10.1136/ annrheumdis-2021-221965
The COVID-19 pandemic has significantly impacted the care and personal lives of people with rheumatic and musculoskeletal diseases (RMDs). Vaccination against COVID-19 has brought optimism and hope but has also raised questions, especially for people with inflammatory RMDs and those receiving drugs that may influence their immune system. To address these questions, EULAR has formed a Task Force of representatives of its constituents, patients, health professionals and rheumatologists experienced in the field.

This Task Force based its advice on knowledge available in November 2021, acknowledging that there is currently limited data about the performance of the different COVID-19 vaccines in patients with RMDs and in patients treated with drugs that influence the immune system. When you read this information, please bear in mind that this text will need to be updated, as new information becomes available.

Several different vaccines are used in national vaccination programmes. All of the vaccines presently being used for COVID-19 are non-live vaccines. They cannot give you the viral disease itself, nor can they transfer infection to you, or change your genetic information, nor is there any evidence that the vaccine imposes a risk to an unborn child. These vaccines have been shown to be safe in people with RMDs as well as in people receiving drugs that influence the immune system. In other infectious diseases (such as influenza), non-live vaccines have been proven to work for immune-suppressed patients. Put simply, there is no reason to withhold these vaccines from patients with RMDs and patients treated with drugs that influence the immune system.

There are a large number of vaccines under development, that work in slightly different ways. Some are being used on a large scale, these have been approved by regulatory bodies such as the World Health Organisation (WHO) and/or European Medicines Agency (EMA) or Food and Drug Administration (FDA). The use of all vaccines worldwide is regulated by local health authorities. In table 1, some more detailed info is given.

Vaccinations should ideally be given when the RMD is in a quiet phase (sometimes referred to as low disease activity or remission); it is also preferable to vaccinate before planned immunosuppression if this is being given intermittently. But of course, this is not always possible during a pandemic. Although it is suggested that vaccination is most effective when the degree of immunosuppression is low, pausing or reducing immunosuppression may increase the risk of flare, and therefore, it is generally advised not to, or only temporarily, interrupt or decrease your medication for this purpose (if you are receiving rituximab, please consult your rheumatologist).

\section{WHEN IS VACCINATION LESS EFFECTIVE IN IMMUNOSUPPRESSED RMD PATIENTS?}

The answer to this important question is based on studies that measured antibody responses to the vaccine in larger groups of RMD patients. Available data (for more details, see table 2) indicate that the immunosuppressive drugs rituximab, cyclophosphamide, mycofenolatemofetil (MMF), abatacept or prolonged use of $10 \mathrm{mg}$ or more prednisone/ daily may decrease the response to the vaccine. In most countries, it is therefore advised that patients using these drugs should receive a third vaccination, at least 1 month after the second vaccination, as part of the initial vaccination cycle to maximise the vaccine response.

This third injection of the vaccine, perhaps better called the third primary dose, has to be seen as part of the initial vaccination cycle. It is different from the so-called 'booster' vaccination, which confusingly, is also called a third vaccination. A booster vaccination may be intended for everyone who completed the primary vaccine series, especially since there is accumulating evidence that the immunity conferred by the vaccine may wane over time. This booster is designed to reinforce the level of immunity to the virus. Many countries have already started a booster vaccination programme. Of course, in specific cases you and your physician can make other choices, based on your personal condition and/or on the drugs you are using; if you are in doubt, consult your rheumatologist.

In addition to the COVID-19-vaccination, we highly recommend vaccination against Pneumococcus and Influenza in patients with RMDs and patients treated with drugs that affect the immune system. (For other vaccinations please consult the current EULAR recommendations on vaccinations: Furer et al, ARD 2020; 79: 39-52; lay version on: https://eular.org/myUploadData/files/vaccination summary_good_for_print_final.pdf).

\section{Frequently asked questions by patients with RMDs and patients using drugs that influence the immune system}

Do I need to be vaccinated? Yes, we encourage everybody to be vaccinated against COVID-19. It 


\begin{tabular}{lll}
\hline $\begin{array}{l}\text { Table } 1 \\
\text { European countries }\end{array}$ & Approved vaccinations, used in at least 5 different \\
\hline Type of vaccine & Pharmaceutical company & Vaccine name \\
\hline Inactivated virus & Sinopharm & BBIBP-CorV \\
\hline & Sinovac & CoronaVac \\
\hline Protein / protein subunit & & Not yet used \\
\hline mRNA & Moderna & mRNA-1273 \\
\hline & BioNTech/Pfizer & BNT162b2 \\
\hline Non-replicating factor & $\begin{array}{l}\text { Johnson\&Johnson } \\
\text { Oxford-Astra Zeneca } \\
\text { Covishield (based on } \\
\text { Oxford-AZ) }\end{array}$ & Ad26.CoV2.s \\
\hline & Serum Institute of India \\
\hline
\end{tabular}

Table 2 Immunosuppressive drugs that might influence the immune response to COVID-19 vaccinations. (See also dgrh.de/Start/ Wissenschaft/Forschung/Covid-19) The advised third vaccination is part of the initial cycle of vaccination, and doesn't refer to the booster vaccination

\begin{tabular}{|c|c|c|}
\hline Name of the drug & $\begin{array}{l}\text { Reduced antibody } \\
\text { response to COVID-19 } \\
\text { vaccination; (effect on } \\
\text { protection unknown) }\end{array}$ & Recommendation \\
\hline Rituximab & Yes & third vaccination advised \\
\hline Mycofenolate mofetil & Yes & third vaccination advised \\
\hline prednisone & Yes, in some circumstances & $\begin{array}{l}\text { When used for a } \\
\text { prolonged period in } \\
\text { dosage of } 10 \mathrm{mg} / \text { day or } \\
\text { higher: third vaccination } \\
\text { advised }\end{array}$ \\
\hline methotrexate & Possibly mild & $\begin{array}{l}\text { No data available, } \\
\text { but consider third } \\
\text { vaccination when } \\
\text { dosage }>20 \mathrm{mg} / \text { week }\end{array}$ \\
\hline Abatacept & Possibly yes & third vaccination advised \\
\hline $\begin{array}{l}\text { JAK-inhibitors (baricitinib, } \\
\text { filgotinib, tofacitinib, } \\
\text { upadacitinib) }\end{array}$ & Possibly yes & third vaccination advised \\
\hline azathioprine & Not known & $\begin{array}{l}\text { No data available, } \\
\text { but consider third } \\
\text { vaccination when } \\
\text { dosage }>2 \mathrm{mg} / \mathrm{kg} / \text { day }\end{array}$ \\
\hline Cyclophosphamide & Not known & third vaccination advised \\
\hline leflunomide & Not known & $\begin{array}{l}\text { No data available, } \\
\text { but consider third } \\
\text { vaccination when } \\
\text { dosage }>20 \mathrm{mg} / \text { day }\end{array}$ \\
\hline
\end{tabular}

NB: Current available evidence suggests that the following medications have no or little influence upon the efficacy of COVID-19 vaccination.

Conventional synthetic antirheumatic drugs: hydroxychloroquine, sulfasalazine, apremilast, tacrolimus, or lower dosages of azathioprine $(2 \mathrm{mg} / \mathrm{kg} /$ day or less), methotrexate ( $20 \mathrm{mg} /$ week or less), leflunomide (20 mg/week or less) and ciclosporine (2 $1 / 2 \mathrm{mg} / \mathrm{kg} /$ day or less).

Biologicals such as the TNFalpha-blockers (adalumimab, certolizumab, etanercept, golimumab, infliximab) the inhibitors of IL-6R (sarilumab, tocilizumab), IL-17A (secukinumab, ixekizumab), IL-12/23 (ustekinumab), IL-23 (guselkumab), IL-1 (canakinumab), IL-1R (anakinra), IL-4 (dupilumab), IL-5 (mepolizumab) and antiBLYSS (belimumab). These biologicals are more modulating than suppressing the immune system. is widely thought that only by vaccinating we may we contain the pandemic.

Do I need to get a third (supplemental) vaccination? Based on scientific evidence a number of RMD patients will need a third vaccination as part of their initial vaccine cycle: see the list in table 2 .

Do I need to get a booster vaccination? In many countries, people are now receiving booster vaccinations as part of strategies to contain the pandemic. It is advised to adhere to the national guidelines.

Is one vaccine better for me than another one? Based on available data no advice can be given for one vaccine over another for patients with RMDs. There are no large studies comparing vaccines, looking at efficacy and safety specifically in patients with RMDs. In many countries not all vaccines are available and national guidelines determine which vaccine can be given. Vaccination, using any of the available, approved vaccines, is definitely better than no vaccination.

What about vaccines that are not listed in table 1 . This list is based on widely approved vaccines. For example, Sputnik V is a non-replicable vector vaccine from Gamaleya; it is approved by local health authorities of some European countries.

Can I get COVID-19 and influenza vaccinations together? Yes, they can be given together, but it is no problem when they are given at different times.

I had COVID-19 and recovered from it. Should I be vaccinated? Yes, vaccination after COVID-19 is safe and provides significant additional protection. In many countries one instead of two vaccinations are given, usually 2-6 months, after recovery from COVID-19.

Can I get the vaccination if I take antirheumatic or immunosuppressive drugs? Yes, you can. There is no danger in receiving the vaccination. The main question is whether the vaccination is effective enough. If you are using immunosuppressive drugs, please consult your rheumatologist about possible decreased efficacy (see also table 2).

Do vaccines interfere with my medication? No.

Do I need to measure my antibody response after vaccination? This is being done for research purposes in groups of patients to collect scientific data to guide clinical practice. This is not recommended in routine clinical care for individual patients, largely because it is unknown which level of antibodies predicts protection against getting infected.

Who should I consult before vaccination-my General Practitioner or my rheumatologist? GPs will be able to answer some of your questions, but for specific questions your rheumatologist should be able to help.

What data are necessary to take the right decision? Knowledge of your disease activity, drug treatment and possible comorbidities.

What about side effects? The approved, available vaccines are remarkably safe, with a similar side effect profile to the influenza vaccination. Based on the reported rare side effects, different countries use different age group rules for different vaccines. This is not related to having an RMD or not; these rules are for everybody. It is advised to adhere to the national guidelines.

What should I do in case of a flare? Luckily, the rates of flares reported in RMDs after COVID-19 vaccination is the same as the rates of flares reported in RMD patients when they are not getting vaccinated. A flare would not likely be related to the vaccine itself, but should you experience any flare for any reason, we recommend you contact your rheumatologist.

What should I do if I have side effects that last longer than 48 hours? This is unlikely, but contact your rheumatologist. 
Will I need a vaccination annually as with other vaccinations for example, influenza? This is unknown for the moment, but it could very well be the case in the future.

What about long-term effects? The evidence so far suggests that, like other vaccines, COVID-19 vaccines are safe short term as well as long term. In contrast, not only can COVID-19 infection cause severe illness in the short term, but so-called 'long COVID-19' can cause severe symptoms over many months.

Am I more at risk of getting COVID-19 infection? No there is no evidence that the risk of getting the infection is higher in patients with RMDs.

Am I more at risk of getting severe COVID-19 infection? Not by your disease itself; but -like in everybody-when you have accompanying medical problems (such as chronic lung disease) or major organ damage (such as kidney problems), the risk can be higher.

Do my treatments increase the risk of severe COVID-19 infection? Most of the drugs used in RMDs have not been associated with severe infection. To date, the only treatments that have been shown to be associated with a severe COVID-19 outcome are rituximab, cyclophosphamide, MMF or using more than $10 \mathrm{mg}$ glucocorticoids daily. Regarding other drugs used in RMDs, we do not have evidence that they are associated with severe COVID-19 infection. Importantly, more active disease is associated with severe outcomes related to infections, including COVID-19. In case you are using one of those drugs mentioned, talk to your rheumatologist about the best options for your situation.
Should I encourage my relatives and friends to get vaccinated? Absolutely, that's the only way to protect each other and contain the pandemic.

Am I fully protected against COVID-19 when I'm vaccinated? Unfortunately, no; you still need to adhere to the general rules: keep distance, wash your hands, ventilate rooms, avoid large groups, self-isolate if you have symptoms, etc.

Collaborators This statement was formulated by the EULAR Taskforce on COVID-19, and the EULAR Recommendations group on COVID-19. Members: Hans Bijlsma (chair), Alessia Alunno, Gerd Burmester, Roberto Caporali, Loreto Carmona, Bernard Combe, Richard Conway, Jeffrey Curtis, Ori Elkayam, Laure Gossec, Lukas Haupt, Marloes Heijstek, Annamaria lagnocco, John Isaacs, Istvan Juhasz, Feline Kroon, Robert Landewe, Pedro Machado, Souzi Makri, Xavier Mariette, lain McInnes, Puja Mehta, Ulf Muller-Ladner, Aurelie Najm, Victoria Navarro-Compan, Julia Rautenstrauch, Diana Rodrigues, Hendrik Schulze-Koops, Josef Smolen, Tanja Stamm, Thea Vliet Vlieland, Dieter Wiek and Kevin Winthrop.

Contributors This viewpoint is submitted on behalf of an EULAR Task Force; the names of all contributors are mentioned above.

Funding The authors have not declared a specific grant for this research from any funding agency in the public, commercial or not-for-profit sectors.

Competing interests None declared.

Patient and public involvement Patients and/or the public were involved in the design, or conduct, or reporting, or dissemination plans of this research. Refer to the Methods section for further details.

Patient consent for publication Not applicable.

Ethics approval This study does not involve human participants.

Provenance and peer review Not commissioned; externally peer reviewed.

ORCID iD

Johannes WJ Bijlsma http://orcid.org/0000-0002-0128-8451 